\title{
Effect of concentric and eccentric porous layer on forced convection heat transfer and fluid flow around a solid cylinder
}

\section{Alireza Alinezhad ( $\sim$ alinezhadalireza1391@gmail.com )}

Department of Chemical Engineering, Faculty of Engineering, Shahid Bahonar University of Kerman, Kerman, 7618868366, Iran

\section{Ataallah Soltani Goharrizi}

Department of Chemical Engineering, Faculty of Engineering, Shahid Bahonar University of Kerman, Kerman, 7618868366, Iran

\section{Ataallah Kamyabi}

Department of Chemical Engineering, Faculty of Engineering, Shahid Bahonar University of Kerman, Kerman, 7618868366, Iran

\section{Research Article}

Keywords: Porous media, Darcy number, Volume averaged equation, eccentricity, Cross-flow

Posted Date: December 1st, 2021

DOI: https://doi.org/10.21203/rs.3.rs-1121969/v1

License: (1) (i) This work is licensed under a Creative Commons Attribution 4.0 International License. Read Full License 


\title{
Effect of concentric and eccentric porous layer on forced convection heat transfer and fluid flow around a solid cylinder
}

\author{
Alireza Alinezhad*, Ataallah Soltani Goharrizi*, Ataallah Kamyabi \\ Department of Chemical Engineering, Faculty of Engineering, Shahid Bahonar University of \\ Kerman, Kerman, 7618868366, Iran \\ * Corresponding author: E-mail: alinezhadalireza1391@gmail.com
}

\begin{abstract}
In this paper, heat transfer and fluid flow around a solid cylinder wrapped with a porous layer in the channel were studied numerically by computational fluid dynamics (CFD). The homogeneous concentric and eccentric porous medium round a rigid, solid cylinder are supposed at local thermal equilibrium. The transport phenomena within the porous layer, volume averaged equations were employed, however the conservation laws of mass, momentum and energy were applied in the channel. This current numerical analysis, the effects of eccentricity $\left(e_{p}=0.005,0.01,0.015,0.02\right)$, the variable diameter of porous layer $(\mathrm{d}=0.07,0.08,0.09)$, permeability, as well as the different Reynolds number and Darcy number on the heat transfer parameters and fluid flow was investigated. The main purpose of this study is analyzed and compared the heat flux of concentric and eccentric porous layer in Reynolds number range of 1 to 40 and Darcy numbers of $10^{-2}$ to $10^{-6}$. It is found that with the decline of Darcy number, the vortex length is increased behind the solid cylinder surface. In addition, the heat flux rate of the cylinder is raised with the increase of Reynolds number. Finally, The results have demonstrated that with raising Reynolds and Darcy numbers, the increase of the average Nusselt numbers in the eccentric porous layer is higher than the concentric porous layer.
\end{abstract}

Keywords: Porous media, Darcy number, Volume averaged equation, eccentricity, Crossflow 


\section{Introduction}

In the past few decades, the variety of numerical studies have been investigated on the convective heat transfer and fluid flow in the porous-coated channel and cylinder surfaces for concentric and eccentric configurations. The behavior of fluid flow in the porous medium is very remarkable because of its unique structure and applications in the various fields such as the design of heat exchangers, energy storage units, fuel cells, thermal management in the cooling of electronic equipment, and improvement of the performance of heat transfer systems. For this reason, numerical and experimental studies have been carried out on this phenomenon.

Many studies have been published about the fluid flow and heat transfer rate in various porous media by a number of researchers. For example, Bhattacharyya et al. [1] were numerically studied fluid flow inside and over a porous layer around solid cylinder surface within Reynolds number range of 1-40 and the porosities of 0.629-0.999. They found that by declining porosity and increasing Reynolds number, the separation point (the separation angle) on the porous layer around solid cylinder surface could be decreased. Bhattacharyya and Singh [2] also numerically offered heat transfer from a porous layer with high conductivity coefficient and considered porosity around a solid cylinder. The optimal amount of porous layer thickness to improve the heat transfer rate was obtained. Their results stated that high porosity layer with high conductivity coefficient $\left(R_{c}=k_{e f f} / k_{f} \geq 5\right)$ could increase the heat transfer rate, even at low permeability $\left(\mathrm{Da}=10^{-5}\right)$. They also found that by increasing Reynolds number, the formation of periodic vortex could be formed. Similarity, Rashidi et al. [3] conducted numerical studies of fluid flow and heat transfer around a cylinder covered with a porous layer. They found that the thermal performance of heat exchanger with high permeability and thermal conductivity of the porous layer was improved. Valipour and Zare Ghadi [4] also numerically simulated the forced convective heat transfer around and through the porous cylinder in Reynolds number range of 1 to 40 and $10^{-6} \leq D a \leq 10^{-2}$. Their numerical results showed that with the rise of Darcy number, the local and average Nusselt numbers were increased. Rong et al. [5] perfomed numerically study a square cylinder covered by a porous layer. They showed that by increasing the porous layer thickness in $D a \geq 10^{-4}$, the amount of drag and lift coefficient was increased and the flow fluid could penetrate into the porous layer easily. Alvandifar et al. [6] numerically investigated a tube bank with 5 rows of tubes wrapped by metal foam layers inside air-cooled 
heat exchangers. Their results showed that heat transfer in the tubes covered with a thin metal foam layer was much more larger than bare tube bank. Another numerically investigation was done by Odabaee et al. [7] for heat transfer rate from a single metal-foam-wrapped solid cylinder in crossflow. Their results showed that heat transfer rate was increased, as a reasult, they realized that pressure drop was increased by raising the porous layer thickness. Rahmati et al. [8] also numerically studied heat transfer rate from a solid cylinder covered with metal foam. They assumed that the local thermal equilibrium between the fluid flow and porous layer surface in the cross-flow of turbulent regime. Their achievement showed that Nusselt number of solid cylinder with a metal porous layer was almost 10 times larger than bar tube without wrapping a metal porous layer. Ait Saada et al. [9] investigated the effects of natural convection on the heat losses and its improvement a horizontal cylinder with fibrous or porou coating. In their reaserch, for the flow movement and heat transfer coefficient were analyzed. According to their numerical results, when the permeability of a limited porous coating have an equivalent to a Darcy number greater than $10^{-6}$ and then the conduction and convection theory combined with the non-Darcian model should be applied. Al-Sumaily et al. [10] carried out numerically study to investigate the forced convection heat transfer from packed bed of spherical particles were accommodated in circular cylinder. They concluded that using a porous medium with large particle diameters were increased heat transfer and decreased the pressure drop. Pedras and Lemos [11] developed turbulent models for fluid flow in a porous medium in two various ways. The first one was using the macroscopic equations employing the extended Darcy-Forchheimer model and the second one was Reynolds-averaged equations. Chen et al. [12] investigated heat transfer of fluctuating tube bundles wrapped with metallic foam, experimentally. According to this experimental study, they concluded that Nusselt number was raised gradually with increasing the amount of fluid flow permeability because of high of porosity in the porous layer. In another experimental study, Al-Salem et al. [13] investigated the effect of porosity and thickness of aluminum porous layer in order to improving heat transfer performance of heated horizontal cylinder with constant heat flux. In this experimental research, they concluded that, the average Nusselt number was increased with raising the porosity of aluminum layer and also with addition of porous layer, the amount of pressure loss was decreased. Calmidi and Mahajan [14] as well as Zhao et al. [15] carried out numerical and experimental analysis on the forced convection in high porosity metal foams. Thermal spread affected in increasing heat transfer. In additionally, this study had shown 
that pore density and various materials could affect on heat transfer and pressure drop. Recently, Ebrahimi et al. [16] analyzed heat transfer characteristics of elastically-mounted solid cylinder covered with concentric porous layer numerically. In their research, the range of various diameter $(\mathrm{d}=0.20,0.5,1)$ and Darcy numbers $\left(10^{-6} \leq D a \leq 10^{-2}\right)$ were considered, respectively. Their simulation showed that by increasing the porous layer diameter of solid cylinder displacement domain, the amount of average Nusselt number was declined. Keshtkar and Dadkhodazadeh [17] investigated the effects of porous gas heat exchanger on fluid flow and heat transfer for two symmetric and asymmetric with the regulation of barriers in cross-flow state by lattice Boltzmann method (LBM). In their study, results show that with increases in Darcy number, permeability within porous gas heat exchanger (PGHE) and natural convective heat transfer rate were raised, respectively. Also, analyzing arrangement of barriers indicated that convective heat transfer rate in asymmetric arrangement was better than symmetric arrangement.

The review of various articles show that most of them have been considered heat transfer and fluid flow around a cylinder covered by a concentric porous layer. The main purpose of this research, investigating the effects of eccentric porous layers with different diameter on heat transfer rate and laminar fluid flow. In order to achieve the objectives of this study, parameters such as the permeability of the eccentric porous layer thickness, the length of recirculation region, tangential and radial velocity, temperature distribution on the surface of rigid and porous layer, heat transfer flux, and the average Nusselt number in the range of different Darcy and Reynolds numbers have been investigated.

\section{Problem Definition}

\subsection{Physical model}

the geometry of problem consists of long, rigid, solid cylinder was covered with a concentric and eccentric porous layer in the channel shows in Fig. 1, respectively. In this current research, The problem was modeled two-dimensional (2-D). To decrease the effects of channel walls on the flow near the solid cylinder, the ratio of channel height (W) to the diameter (D) of solid cylinder must be high $\left.\frac{W}{(D}=\frac{0.5}{0.05}=10\right)$, therefore, we ignored it in this study. We considered the 
constant temperature of solid cylinder surface at $313 \mathrm{~K}$ and the temperature of inlet fluid flow was fixed at $293 \mathrm{~K}$. Also, the diameter of solid cylinder is $0.05 \mathrm{~m}$ and the characteristic of channel is $0.5 \times 1 \mathrm{~m}$. The boundary conditions included no-slip at adjacent surface of channel (upper and lower surface) and the solid cylinder surface. A laminar flow was considered to run around the isothermal cylinder. The porous zone around the cylinder was assumed to be homogeneous and isotropic. All fluid properties were considered to be constant. The temperature of solid phase was equal to the fluid phase (local thermal equilibrium (LTE)).

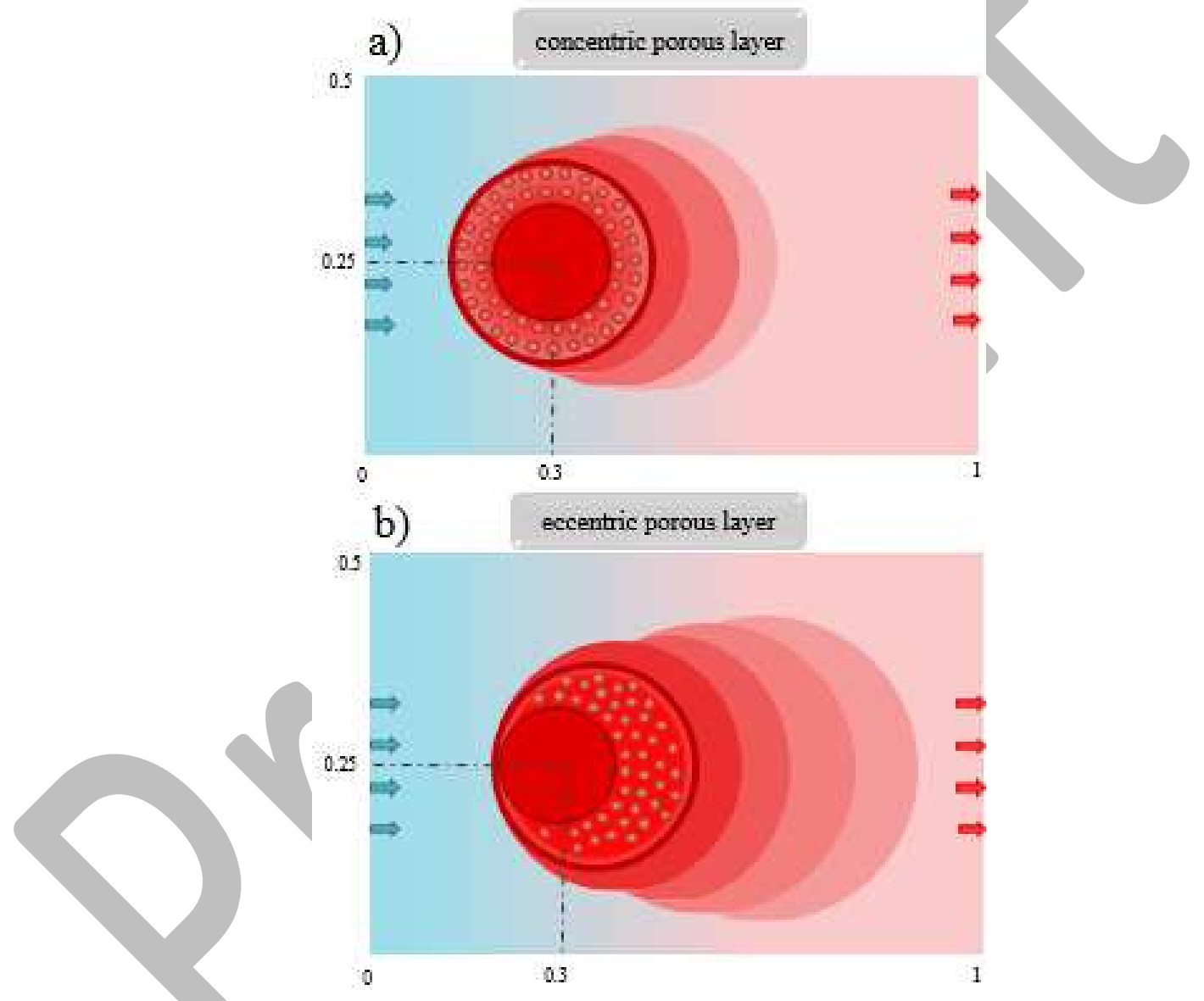

Fig. 1. Schematic of the solid cylindrical geometry; a) concentric porous layer, b) eccentric porous layer.

\subsection{Governing equations}

Equations governing the clear fluid zones are Navier-Stokes equations and those that govern the porous medium zones are the volume averaged equations. The clear fluid and porous medium domains are indicated by the subscripts (1) and (2), respectively.

The mass conservation equation in the region 1 (clear fluid) [3]: 


$$
\frac{\partial}{\partial r}\left(r u_{1}\right)+\frac{\partial}{\partial \theta}\left(v_{1}\right)=0
$$

And the momentum equations in the $\mathrm{r}$ and $\theta$ direction [3]:

$$
\begin{aligned}
& \left(\frac{v_{1}}{\mathrm{r}} \frac{\partial \mathrm{u}_{1}}{\partial \theta}+\mathrm{u}_{1} \frac{\partial \mathrm{u}_{1}}{\partial \mathrm{r}}-\frac{v_{1}^{2}}{\mathrm{r}}\right)=-\frac{\partial \mathrm{p}_{1}}{\partial \mathrm{r}}+\frac{2}{\mathrm{Re}}\left(\frac{\partial^{2} \mathrm{u}_{1}}{\partial \mathrm{r}^{2}}+\frac{1}{\mathrm{r}} \frac{\partial \mathrm{u}_{1}}{\partial \mathrm{r}}+\frac{1}{\mathrm{r}^{2}} \frac{\partial^{2} \mathrm{u}_{1}}{\partial \theta^{2}}-\frac{2}{\mathrm{r}^{2}} \frac{\partial v_{1}}{\partial \theta}-\frac{\mathrm{u}_{1}}{\mathrm{r}^{2}}\right) \\
& \left(\frac{v_{1}}{\mathrm{r}} \frac{\partial v_{1}}{\partial \theta}+\mathrm{u}_{1} \frac{\partial v_{1}}{\partial \mathrm{r}}+\frac{\mathrm{u}_{1} v_{1}}{\mathrm{r}}\right)=-\frac{1}{\mathrm{r}} \frac{\partial \mathrm{p}_{1}}{\partial \theta}+\frac{2}{\mathrm{Re}}\left(\frac{\partial^{2} v_{1}}{\partial \mathrm{r}^{2}}+\frac{1}{\mathrm{r}} \frac{\partial v_{1}}{\partial \mathrm{r}}+\frac{1}{\mathrm{r}^{2}} \frac{\partial^{2} v_{1}}{\partial \theta^{2}}+\frac{2}{\mathrm{r}^{2}} \frac{\partial \mathrm{u}_{1}}{\partial \theta}-\frac{v_{1}}{\mathrm{r}^{2}}\right)
\end{aligned}
$$

The energy equation in the region 1 can be written as [3]:

$$
\left(u_{1} \frac{\partial T_{1}}{\partial r}+\frac{v_{1}}{r} \frac{\partial T_{1}}{\partial \theta}\right)=\frac{2}{R e P_{r}}\left(\frac{\partial^{2} T_{1}}{\partial r^{2}}+\frac{1}{r} \frac{\partial T_{1}}{\partial r}+\frac{1}{r^{2}} \frac{\partial^{2} T_{1}}{\partial \theta^{2}}\right)
$$

In the region 2 (the porous medium region), the volume-averaged mass conservation equation becomes [3]:

$$
\frac{\partial}{\partial r}\left(r u_{2}\right)+\frac{\partial}{\partial \theta}\left(v_{1}\right)=0
$$

And the momentum equations in the $\mathrm{r}$ and $\theta$ direction can be written as [3]:

$$
\begin{aligned}
& \frac{1}{\varepsilon}\left(\frac{v_{2}}{\mathrm{r}} \frac{\partial \mathrm{u}_{2}}{\partial \theta}+\mathrm{u}_{2} \frac{\partial \mathrm{u}_{2}}{\partial \mathrm{r}}-\frac{v_{2}^{2}}{\mathrm{r}}\right)=-\varepsilon \frac{\partial \mathrm{p}_{2}}{\partial \mathrm{r}}+\Lambda \frac{2 \varepsilon}{\mathrm{Re}}\left(\frac{\partial^{2} \mathrm{u}_{2}}{\partial \mathrm{r}^{2}}+\frac{1}{\mathrm{r}} \frac{\partial \mathrm{u}_{2}}{\partial \mathrm{r}}+\frac{1}{\mathrm{r}^{2}} \frac{\partial^{\mathrm{x}} \mathrm{u}_{2}}{\partial \theta^{2}}-\frac{2}{\mathrm{r}^{2}} \frac{\partial v_{2}}{\partial \theta}-\frac{\mathrm{u}_{2}}{\mathrm{r}^{2}}\right) \\
& -\frac{\varepsilon}{2 \mathrm{ReDa}} \mathrm{u}_{2}-\frac{\varepsilon \mathrm{C}_{\mathrm{E}}}{2 \sqrt{\mathrm{Da}}} \sqrt{\mathrm{u}_{2}^{2}+v_{2}^{2}} \mathrm{u}_{2} \\
& \frac{1}{\varepsilon}\left(\frac{v_{2}}{r} \frac{\partial v_{2}}{\partial \theta}+u_{2} \frac{\partial v_{2}}{\partial r}+\frac{u_{2} v_{2}}{r}\right)=-\frac{\varepsilon}{r} \frac{\partial p_{2}}{\partial \theta}+\Lambda \frac{2 \varepsilon}{F e}\left(\frac{\partial^{2} v_{2}}{\partial r^{2}}+\frac{1}{r} \frac{\partial v_{2}}{\partial r}+\frac{1}{r^{2}} \frac{\partial^{2} v_{2}}{\partial \theta^{2}}+\frac{2}{r^{2}} \frac{\partial u_{2}}{\partial \theta}-\frac{v_{2}}{r^{2}}\right) \\
& -\frac{\varepsilon}{2 R e D a} v_{2}-\frac{\varepsilon C_{F}}{2 \sqrt{D a}} \sqrt{u_{2}^{2}+v_{2}^{2}} v_{2}
\end{aligned}
$$

The energy equation with the local thermal equilibrium assumption [3]:

$$
\left(u_{2} \frac{\partial T_{2}}{\partial r}+\frac{v_{2}}{r} \frac{\partial T_{z}}{\partial \theta}\right)=\frac{2 R_{c}}{R \theta P r}\left(\frac{\partial^{2} T_{z}}{\partial r^{2}}+\frac{1}{r} \frac{\partial T_{z}}{\partial r}+\frac{1}{r^{2}} \frac{\partial^{2} T_{z}}{\partial \theta^{2}}\right)
$$

The volume-averaged fluid velocity, $\vec{V}_{s}$ inside the porous region with the porosity $(\varphi=\varepsilon)$ is related to the fluid velocity $(\vec{v})$, through the Dupuit-Forchheimer relationship, as $\vec{v}=\varphi \vec{V}$. Here we used the Ergun correlation to calculate the Forchheimer coefficient, $C_{F_{8}}$ [18]:

$$
C_{F}=\frac{1.75}{\sqrt{150 \varphi^{8}}}
$$

The permeability of the porous medium is calculated from the Kozeny-Carmen relation, as shown below [19]:

$$
K=\frac{d_{p}^{2} \varphi^{s}}{150(1-\varphi)^{2}}
$$


where $\mathrm{K}$ is the permeability and $d_{p}$ is the average particle size in the porous layer.

\subsection{Boundary conditions}

Dimensions of the computational domain should be selected in a way to minimize the effects of the outer boundaries. The size of channel to the two-dimensional computational domain is $0.5 \mathrm{~m} \times 1 \mathrm{~m}$. The governing equations (1) - (8) are subjected to the following boundary conditions.

The hydrodynamic and thermal boundary condition of inlet fluid flow at the entrance of channel:

$$
u=U_{\infty} \quad v=0 \quad T=T_{\infty}
$$

The fluid flow is both hydraulically and thermally fully developed in the $\mathrm{r}$ direction. The hydrodynamic and thermal boundary condition at the upper and lower boundaries are symmetric:

$$
\frac{\partial u_{1}}{\partial r}=0 \quad \frac{\partial v_{1}}{\partial r}=0 \quad T_{1}=T_{\infty}
$$

no-slip wall boundary condition with a fixed wall temperature on the solid cylinder surface:

$$
u_{2}=0 \quad v_{2}=0 \quad T_{2}=T_{w}
$$

Along the upstream boundary (uniform flow):

$$
0<\theta<\frac{\pi}{4} \Rightarrow u_{1}=-\cos \theta, v_{1}=\sin \theta, T_{1}=T_{\infty}
$$

\subsection{Coupling conditions}

In the current study, we assumed the continuity of velocity, shear stress, temperature and heat flux here at the interface between the porous layer and the fluid area:

$$
\begin{gathered}
u_{1}=u_{2}, v_{1}=v_{2} \\
\mu_{f} \frac{\partial v_{1}}{\partial r}=\frac{\mu_{f}}{\varphi} \frac{\partial v_{2}}{\partial r} \\
T_{1}=T_{2} \\
\frac{\partial T_{1}}{\partial r}=\frac{\varphi k_{f}+(1-\varphi) k_{g}}{k_{f}} \frac{\partial T_{2}}{\partial r}, \frac{\partial T_{1}}{\partial \theta}=\frac{\varphi k_{f}+(1-\varphi) k_{g}}{k_{f}} \frac{\partial T_{2}}{\partial \theta}
\end{gathered}
$$

\subsection{Numerical method}

In this paper, numerical investigations were obtained by using the finite volume methodbased solver, The steady, pressure-based solver was chosen to solve the thermo-fluid-dynamic 
problem discussed in this study. In the porous zone, to solve the fluid flow, the energy equations, Darcy-Brinkmann-Forchheimer model and local thermal equilibrium (LTE) was used and activated. SIMPLE algorithm was used to couple the pressure and the velocity terms for the pressure correction equation. The least squares cell-based method was applied to construct the values of a scalar at the cell faces. A second order discretization scheme for pressure and a second order upwind discretization scheme for momentum and energy were considered.

\subsection{Grid independent study}

In order to ensure the simulation results are the independent of the mesh size, the values of the heat flux on the solid cylinder surface wrapped with a eccentric porous layer were calculated,so, we found that the independent of the mesh size was reasenable. Then we choosed the number of cells (76360) to avoid the waste of time and cost in this research. Also, we indicate comparison of heat flux for different grids according to the Reynolds and porosity of 40 and 0.70 in the table 1 , respectively.

\section{Table 1}

Comparison of heat flux on the cylinder surface for different grids.

\begin{tabular}{ccc}
\hline Number of cells & Heat flux $\left(\frac{\mathrm{w}}{\mathrm{w}^{2}}\right)$ & Deviation (\%) \\
\hline 76360 & 23.14 & 0.08 \\
108170 & 23.12 & \\
326875 & 23.04 & 0.08 \\
340900 & 23.06 & \\
\hline & & \\
2.7. Validation & & \\
\end{tabular}

In the first of validation, The numerical data had been compared with the distinct experimental data. The Nusselt number distribution passing through the solid cylinder without a porous layer had been studied experimentally by many reaseches. Fig. 2 shows the investigations of other studies and considered the numerical and experimental data for Nusselt number distribution. Then, in the present study, three various diameters of the rigid layers covered around the solid cylinder in both concentric and eccentric states were considered and compared with the numerical data [20]. Table 2 shows the variable diameter of the layers with the amounts of heat flux in both of concentric and eccentric states. In the final stage of validation, the results of the 
tangential and radial velocity distribution on the surface of concentric porous layer covered around the solid cylinder with Darcy number of $10^{-2}$ had been compared with the numerical results [3]. The results of this comparison for tangential and radial velocities show in Figs. 3 and 4 , respectively.

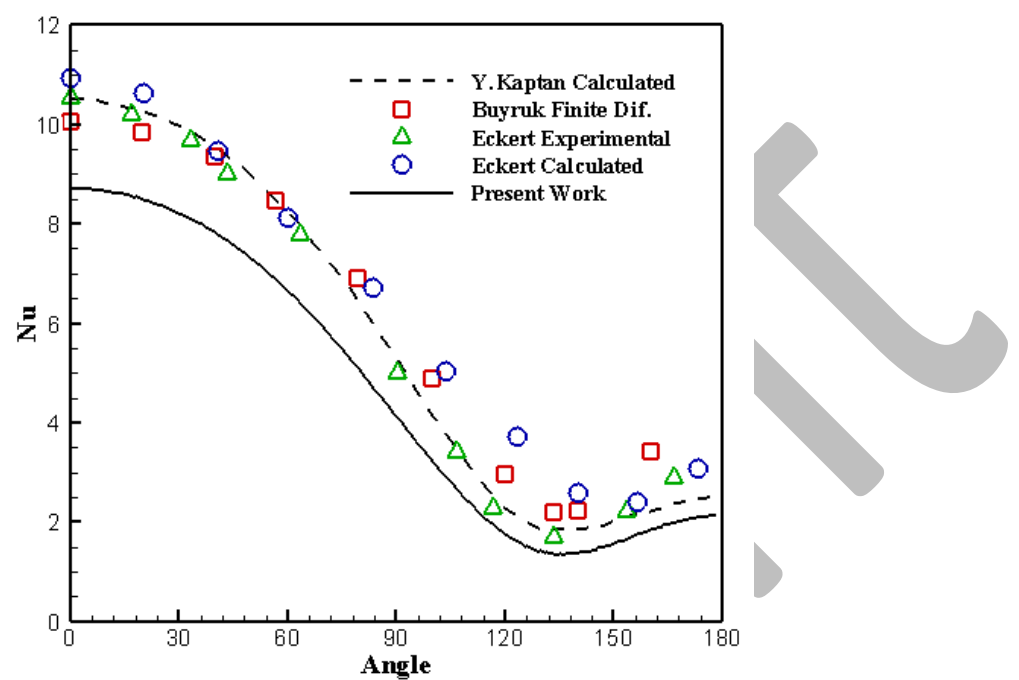

Fig. 2. Distribution of Nusselt number on the clean tube parameter.

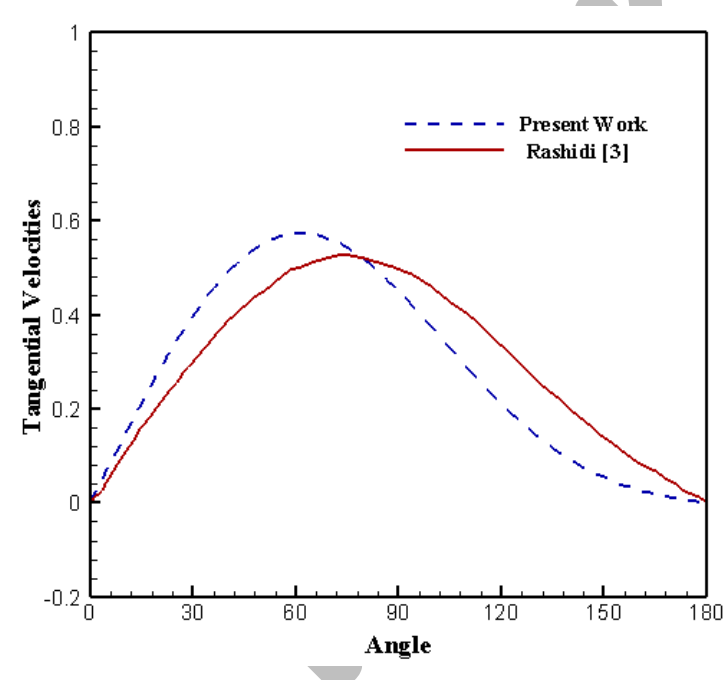

Fig. 3. The comparison of tangential velocity distribution on the porous layer with numerical results.

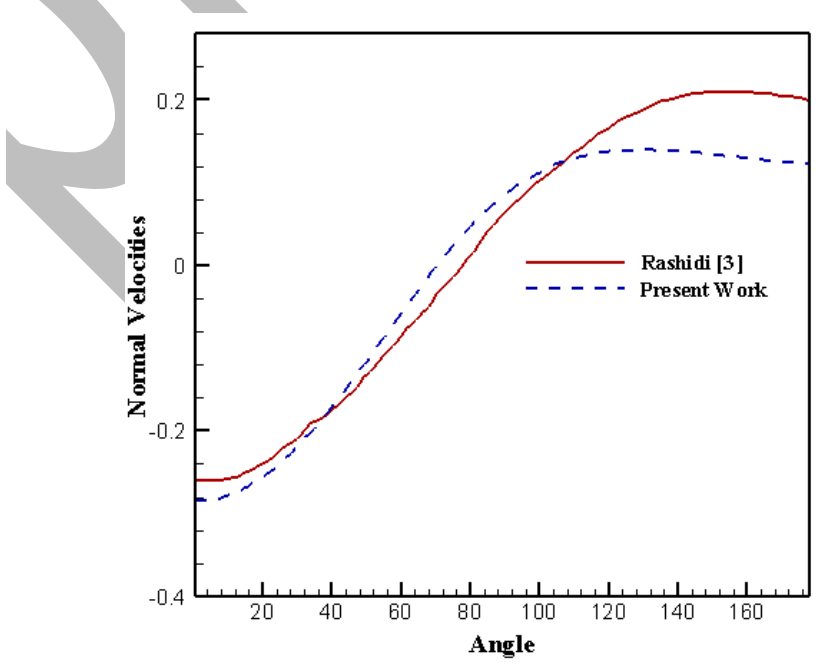

Fig. 4 . The comparison of radial velocity distribution on the porous layer with numerical results. 
Table 2

Numerical validation of the heat flux transfer results.

\begin{tabular}{ccccc}
\hline \multirow{2}{*}{$\begin{array}{c}\text { Type of } \\
\text { symmetry }\end{array}$} & $\begin{array}{c}\text { Diameter } \\
(\mathrm{m})\end{array}$ & \multicolumn{2}{c}{ Heat transfer flux on the surface of the rigid layer $\left(\frac{\mathrm{w}}{\mathrm{m}^{2}}\right)$} & \multirow{2}{*}{\begin{tabular}{c} 
Deviation \\
\cline { 3 - 4 }
\end{tabular}} \\
\cline { 3 - 4 } & 0.07 & Simulation $[20]$ & Simulation (Present Work) & $(\%)$ \\
\hline Concentric & 0.07 & 32.13 & 30.60 & 4.7 \\
Eccentric & 0.08 & 26.36 & 31.58 & 2.07 \\
Concentric & 0.08 & 27.15 & 25.19 & 4.4 \\
Eccentric & 0.09 & 22.14 & 25.90 & 4.6 \\
Concentric & 0.09 & 22.82 & 21.30 & 4.2 \\
Eccentric & & & 21.82 & 4.3 \\
\hline
\end{tabular}

\section{Results and discussion}

\subsection{Hydrodynamic results}

Fig. 5 shows the streamlines of fluid flow with $R e=1$ and $R e=40$ passing through eccentric porous layer and the solid cylinder. In this case, the streamlines go curved surrounding solid cylinder surface and the porous layer. In fact, it could be said that the fluid flow was entirely wrapped around the solid cylinder along with the porous layer. However, if the Reynolds number were increased, the separation of the boundary layer took place. Also, the formation of the wake behind the solid cylinder was created.

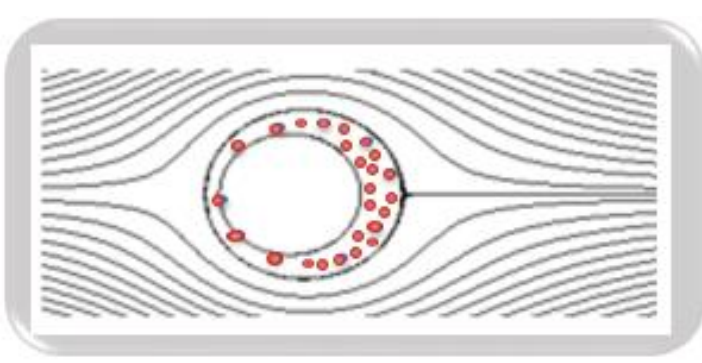

$R e=1$

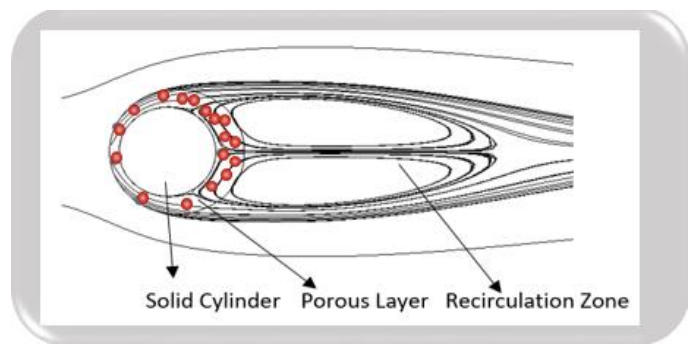

$\operatorname{Re}=40$

Fig. 5. Schematic of the vortexes formed behind the solid cylinder.

In Fig. 6 the effects of the Reynolds number on the streamlines and the length of the vortexes at the behind of the solid cylinder and the outer surface of the eccentric porous layer for all four 
distinct state (a) $D a=10^{-2}$ (b) $D a=10^{-3}$ (c) $D a=10^{-5}$ (d) $D a=10^{-6}$ with the same diameter of the porous layer $(\mathrm{d}=0.07 \mathrm{~m})$ have been shown. According to Darcy number, it could be seen that with the increase of the Darcy number, the amount of the fluid penetration in the porous layer was raised and the fluid flow could transform freely within the porous layer. As shown in Fig. 6, at low Reynolds number $(\operatorname{Re}=1)$, the streamlines in each of the four state $(\mathrm{a}, \mathrm{b}, \mathrm{c}, \mathrm{d}$ at $\operatorname{Re}=1)$ had sticked to the outer porous layer surface, and no vortex was created behind the solid cylinder. Since the Reynolds number was increased $(R e=10,20,30$ and 40), a pair of vortexes were formed behind the solid cylinder and the porous layer. So, in general, it could be seen that by raising Darcy number, the quantity of the fluid penetration in the porous layer was expanded. Similarity, by raising the Reynolds number, the vortex length were developed behind the solid cylinder and the porous layer. Although, it could be concluded that with the decrease of Darcy number, the vortex length is increased, it means that a pair of vortexes stretches from porous layer and into fluid field with the greater Darcy number. Also, the vortexes would be closer to the solid cylinder surface.

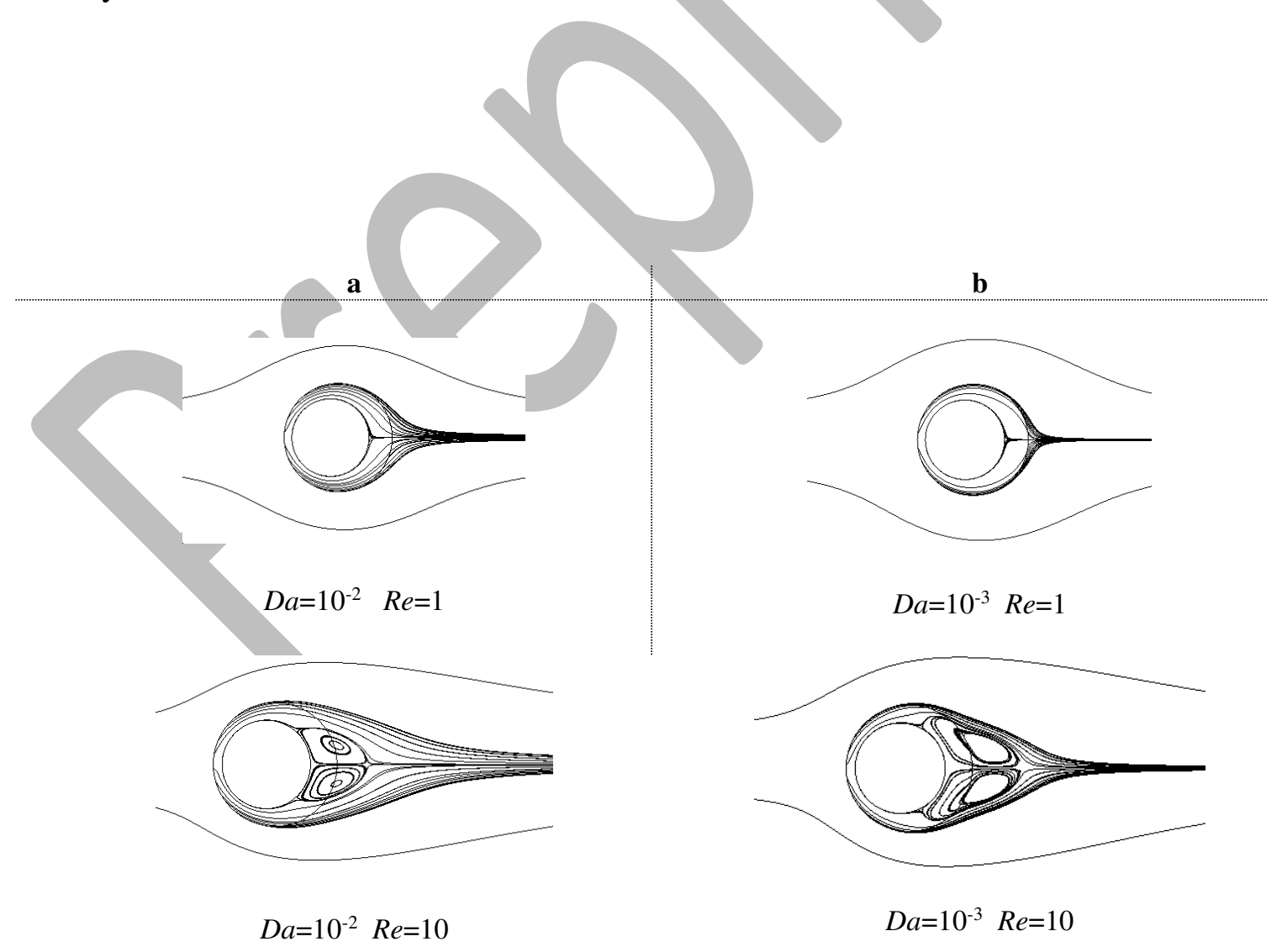




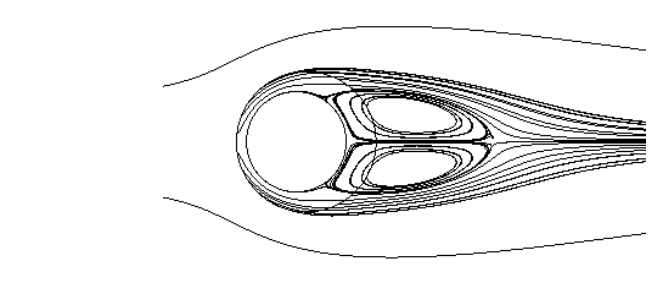

$D a=10^{-2} \quad R e=20$

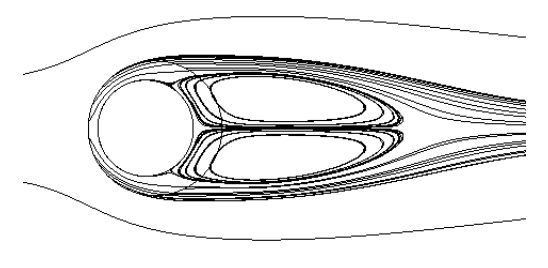

$D a=10^{-2} \quad R e=30$

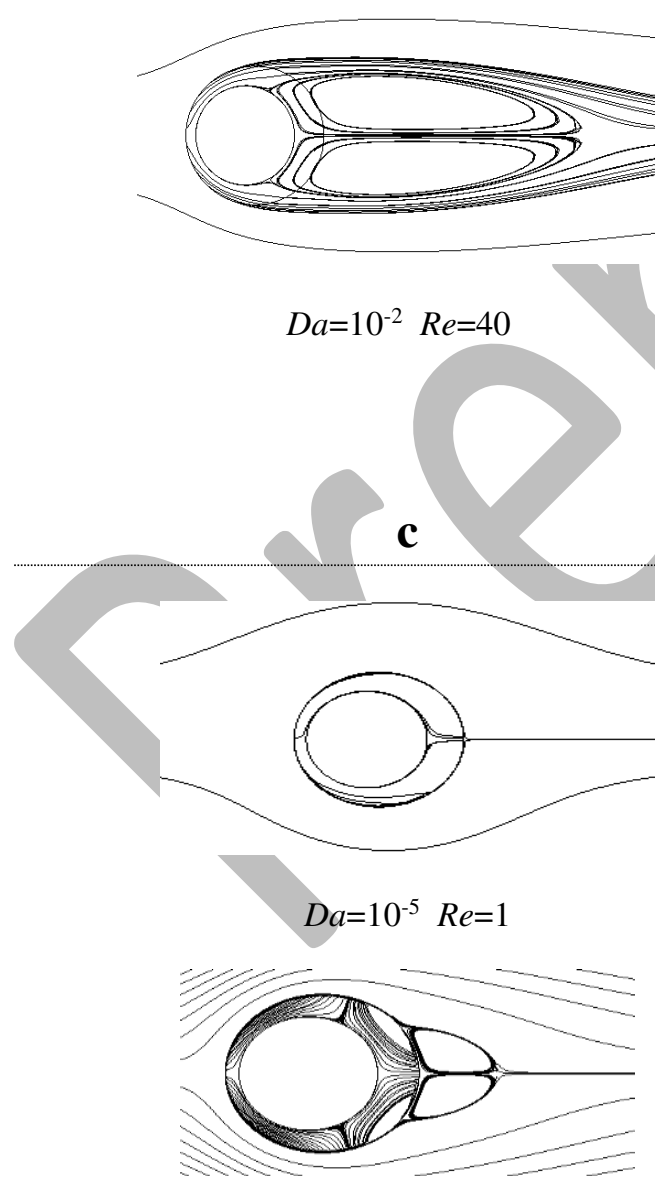

$D a=10^{-5} \quad R e=10$

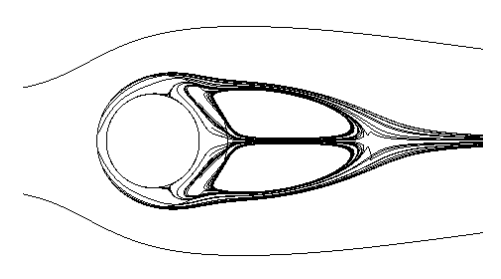

$D a=10^{-3} \quad R e=20$

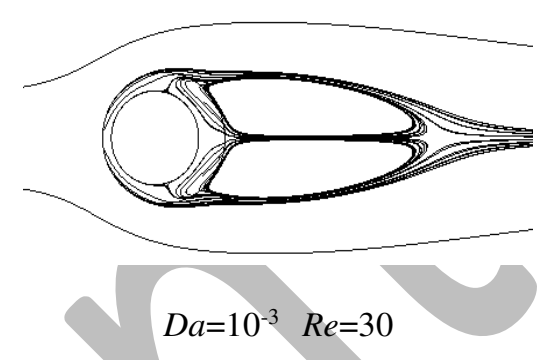

$D a=10^{-3} \quad R e=40$

d

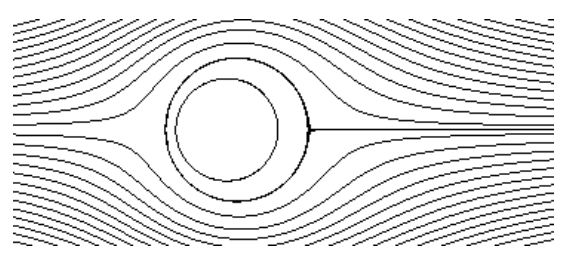

$D a=10^{-6} \quad R e=1$

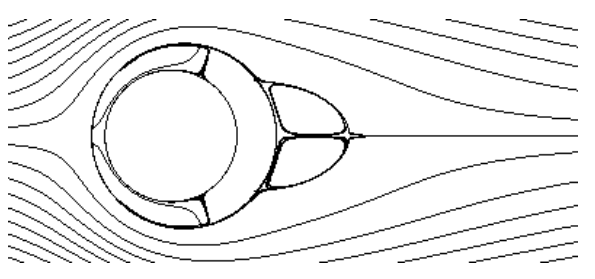

$D a=10^{-6} \quad R e=10$ 

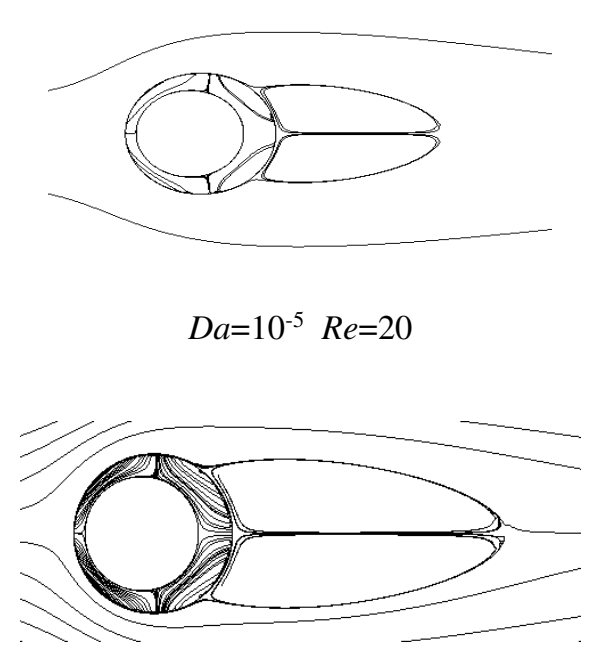

$D a=10^{-5} \quad R e=30$

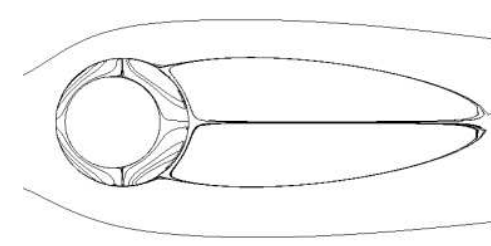

$D a=10^{-5} \quad R e=40$
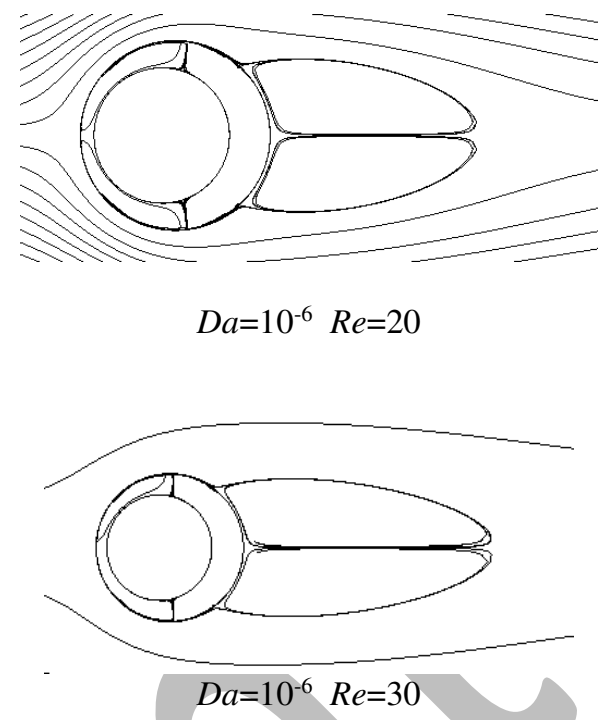

$D a=10^{-6} \quad R e=30$

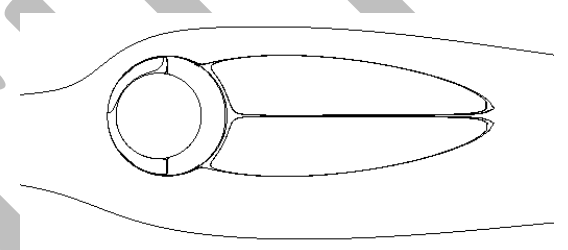

$D a=10^{-6} \quad R e=40$

Fig. 6. Configuration of streamlines in various Reynolds and Darcy numbers.

Figs. 7 and 8 show the effects of Darcy number on the distribution of tangential and radial velocities on the eccentric porous layer surface. As you can see, as Darcy number was increased gradually, the measure of vertical velocities on the eccentric porous layer surface was raised,too. On the other hand, it also seemed that the distribution of tangential velocities in $D a=10^{-2}$ and $D a=10^{-3}$ in the direction of their velocity around an angle of $80^{\circ}$ became in reverse. From both Fig. 8 and Fig. 9, It can be also concluded the porouse layer surface and the rigid layer sarface were identically at lower Darcy numbers becuase of the permeability on the eccentric porous layer surface was decreased. At the result, on the eccentric porous layer surface was created the semi-slip boundary condition and the simulated data of the tangential and normal velocities became approximately zero. 


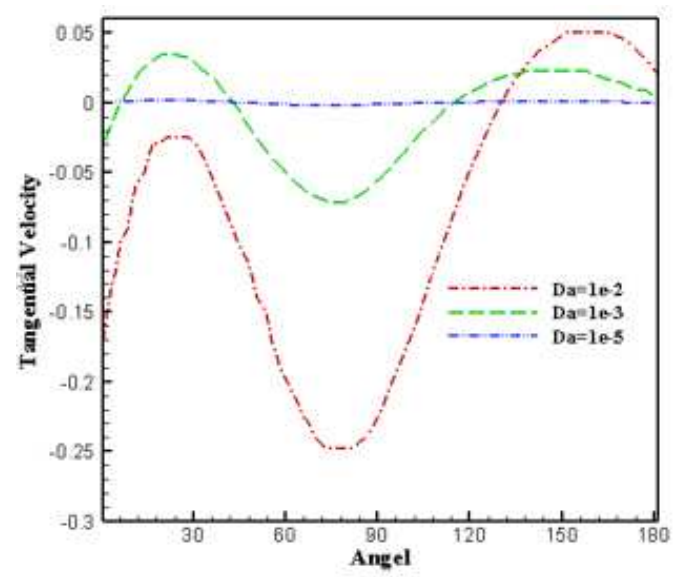

Fig.7. Tangential velocities on the eccentric porous layer surface with a diameter of $0.09 \mathrm{~m}$ and Reynolds 40 .

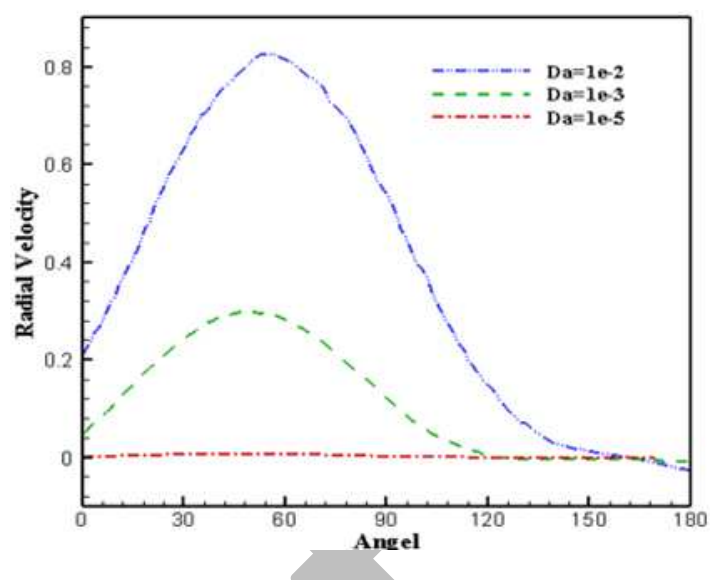

Fig. 8. Radial velocities on the eccentric porous layer surface with a diameter of $0.09 \mathrm{~m}$ and Reynolds of 40 .

\subsection{Heat transfer results}

\subsubsection{Temperature distribution}

Comparison of temperature distribution on the eccentric porous layer with the eccentric rigid layer was conducted and has been shown in Figs. 9 (a) and 9 (b), respectively. The variation in the Reynolds number could change the profile of temperature distribution and temperature contour lines. The results have been shown in Figs. 9 (a) and (b). Fig. 9 (a) shows the temperature distribution around the solid cylinder covered by an eccentric porous layer with $D a=10^{-2}$. Fig.9 (b), displays temperature distribution around the solid cylinder wrapped with an eccentric rigid layer. In both cases, temperature distribution in distinct Reynolds numbers $(1 \leq R e \leq 40)$ and the same diameter was analyzed. We can obtain that the temperature distribution of inner and outer the rigid layer surface was more much more than inner and outer of the porous layer surface. In fact, if the layer surrounding the solid cylinder is considered rigid, the more volume of the fluid flow passing through the layer surface can under the influence of the cylinder temperature.

Fig. 10 has been shows temperature distribution on the different of rigid layer diameter and porous layer diameter with the same Reynolds number $(\operatorname{Re}=20)$. it could be concluded that the temperature distribution on the rigid layer surface was higher than the temperature distribution 
on the porous layer surface, this means that, the conduction heat transfer in the rigid state was much more than the porous state.

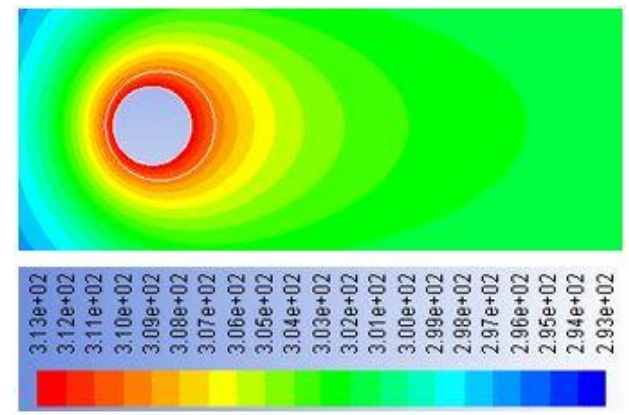

$R e=1$ (a)
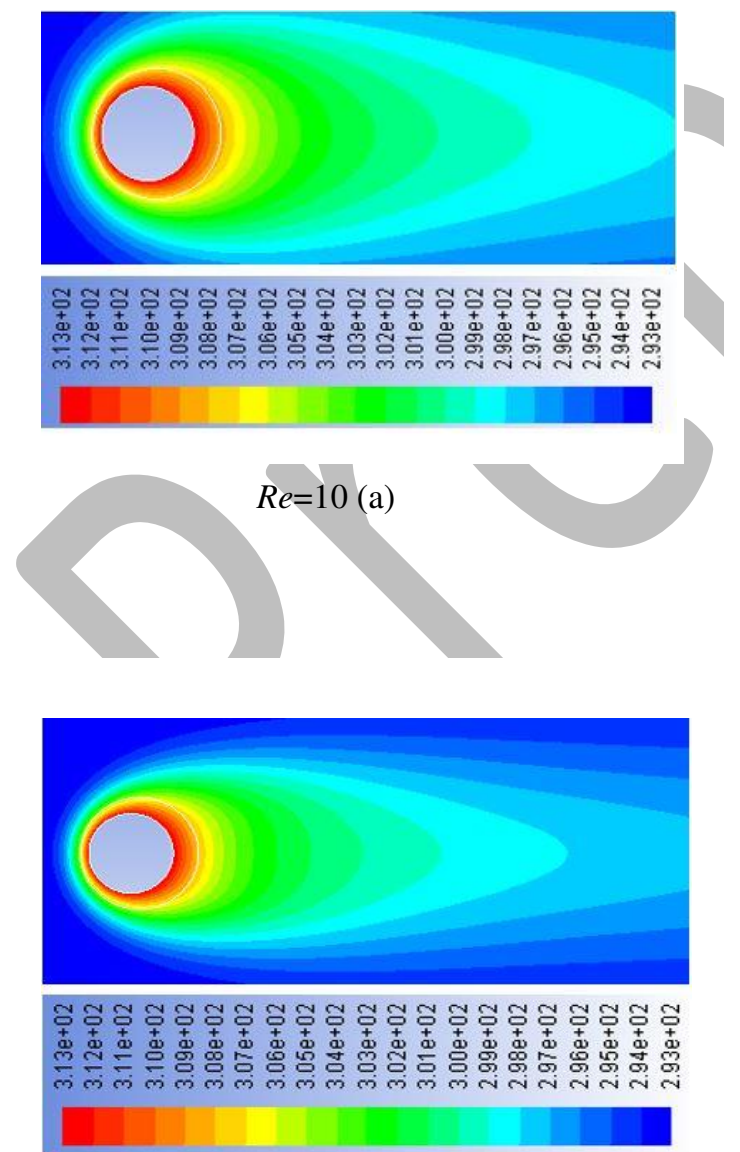

$R e=20$ (a)
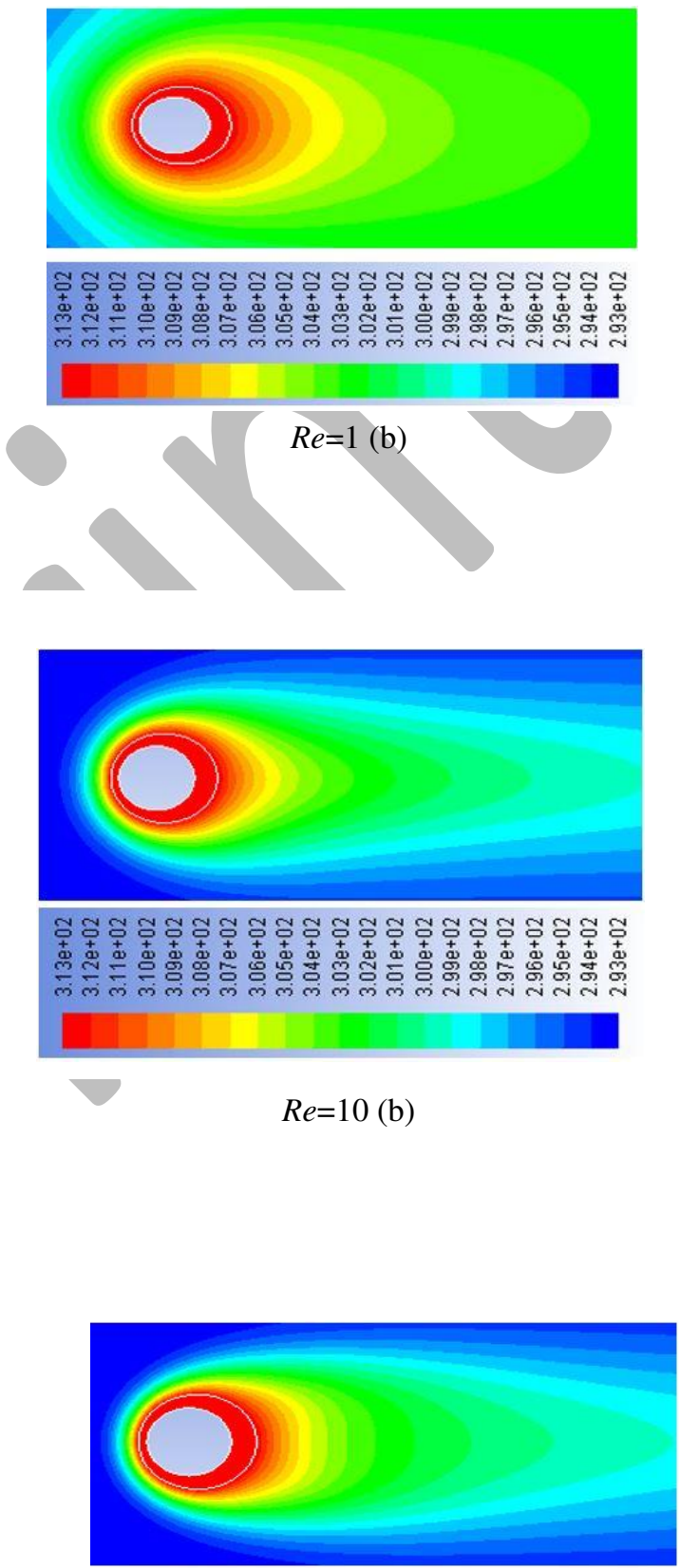

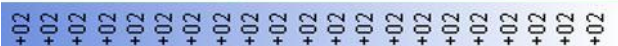

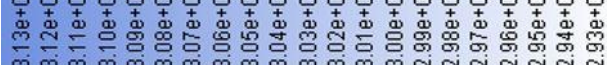
$R e=20(b)$ 


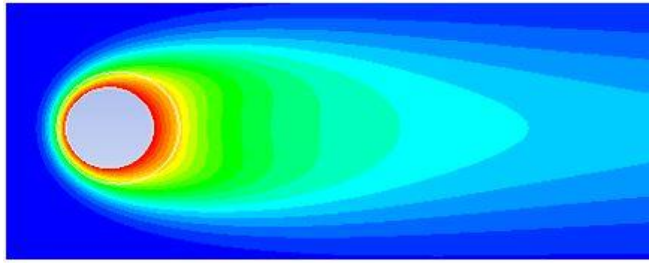

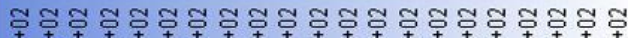
ल $\operatorname{Re}=30(\mathrm{a})$

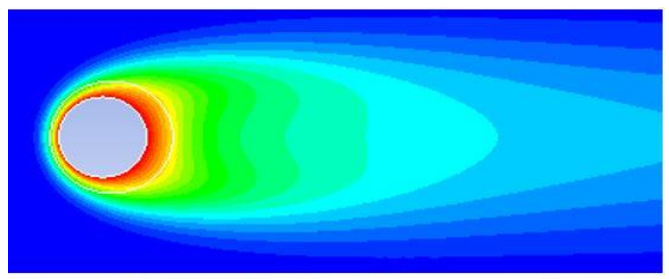

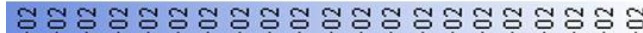

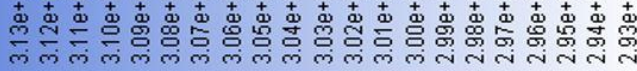

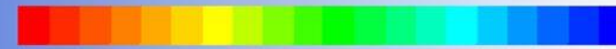

$$
\operatorname{Re}=40(\text { a) }
$$
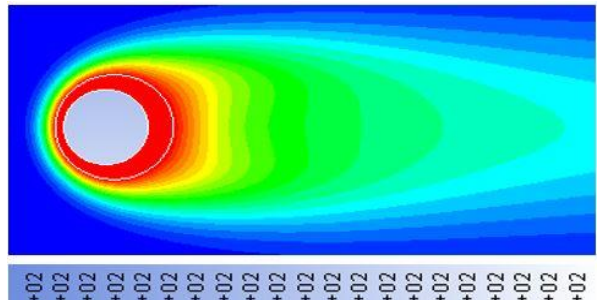

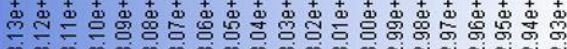

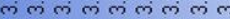
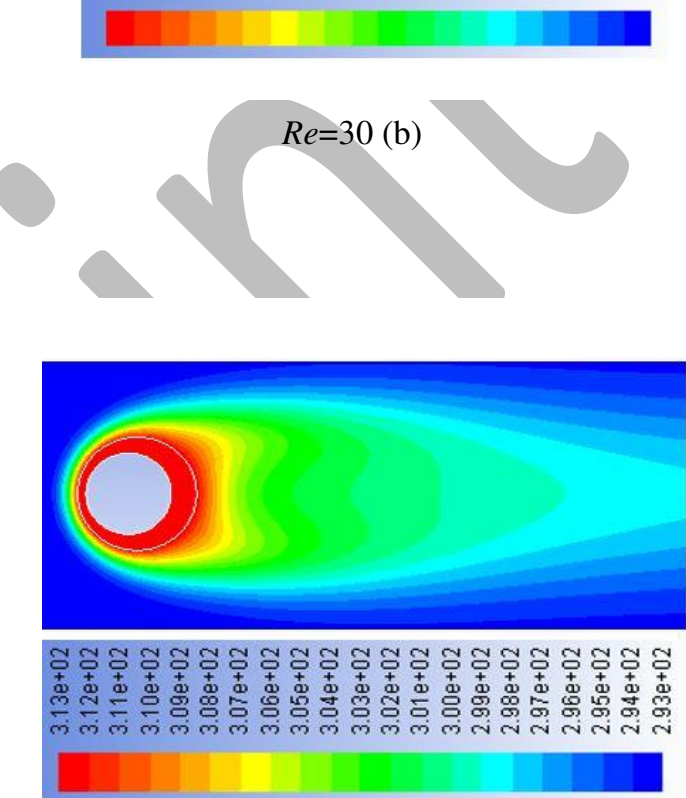

$R e=40(\mathrm{~b})$

Fig.9 (a). The Temperature distribution for an eccentric porous layer with diameter of $0.07 \mathrm{~m}$ and

Fig.9 (b). The temperature distribution for an eccentric $D a=10^{-2}$. rigid layer with diameter of $0.07 \mathrm{~m}$ and $D a=10^{-2}$. 


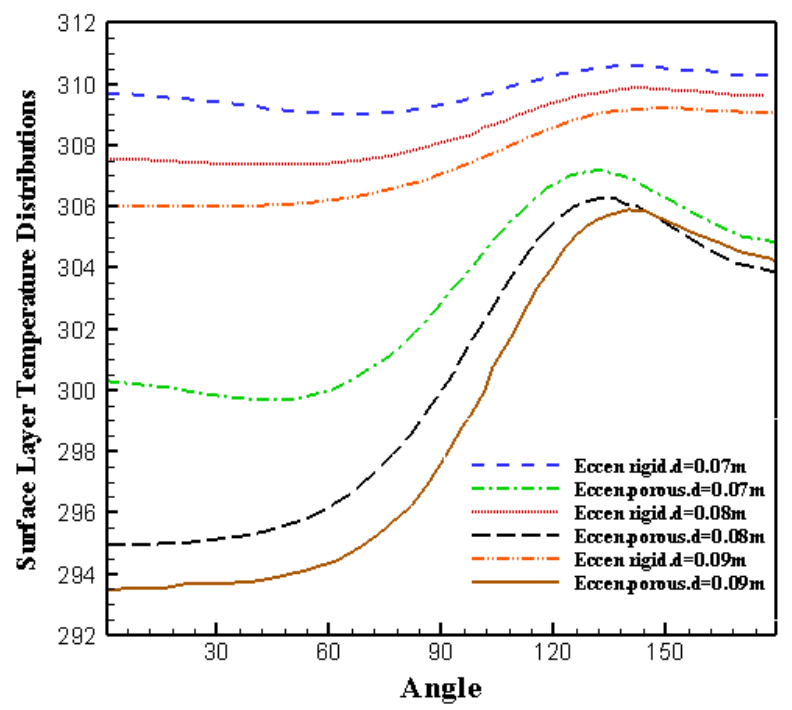

Fig. 10. Distribution of temperature on the surface of rigid and porous layer at $D a=10^{-2}$.

\subsubsection{Heat transfer flux}

The effects of Darcy and Reynolds numbers on the heat transfer flux from the cylinder surface to the diameters of concentric and eccentric porous layer states were accomplished by the simulated methods. Table 3 and Table 4, show the the heat transfer flux from the solid cylinder surface in the various Darcy numbers and Reynolds numbers with different diameters, respectively. The results were obtained by the simulated methods in Table 3 and Table 4 which are as follows:

1- In the solid cylinder with constant wall temperature, by raising the Darcy number, the heat transfer flux through the cylinder could be increased.

2- In the constant Darcy number, with the increase in the diameter of the porous layer, the heat transfer flux passing through the cylinder was dropped.

3- By increasing Reynolds number of the porous layer in a constant diameter, the heat transfer flux of the solid cylinder was raised,too.

4- In the constant Reynolds number, with the increase in the diameter of the porous layer, the heat transfer flux of the solid cylinder was decreased.

5- the main reasult was the amount of the heat transfer flux of the solid cylinder into fluid field in eccentric porous layer state was five percent (5\%) higher than that concentric porous layer state. 
Table 3

Simulated results for the heat transfer flux $\left(\mathrm{w} / \mathrm{m}^{2}\right)$ in $R e=40$.

\begin{tabular}{ccrrrrr}
\hline Type of symmetry & Diameter $(\mathrm{m})$ & $D a=10^{-2}$ & $D a=10^{-3}$ & $D a=10^{-4}$ & $D a=10^{-5}$ & $D a=10^{-6}$ \\
\hline Concentric & 0.07 & 29.94 & 25.25 & 24.06 & 23.85 & 23.82 \\
Concentric & 0.08 & 27.41 & 22.24 & 21.27 & 21.12 & 21.10 \\
Concentric & 0.09 & 25.74 & 20.15 & 19.30 & 19.20 & 19.18 \\
Eccentric & 0.07 & 31.14 & 27.14 & 25.77 & 25.49 & 25.45 \\
Eccentric & 0.08 & 28.45 & 23.49 & 22.40 & 22.23 & 22.21 \\
Eccentric & 0.09 & 26.45 & 21.02 & 20.08 & 19.96 & 19.95 \\
\hline
\end{tabular}

\section{Table 4}

Simulated results for the heat transfer flux (w/m2) in $D a=10^{-2}$.

\begin{tabular}{ccccccc}
\hline Type of symmetry & Diameter $(\mathrm{m})$ & $R e=1$ & $R e=10$ & $R e=20$ & $R e=30$ & $R e=40$ \\
\hline Concentric & 0.07 & 9.22 & 17.64 & 23.42 & 26.91 & 29.94 \\
Concentric & 0.08 & 9.09 & 17.48 & 21.43 & 24.56 & 27.46 \\
Concentric & 0.09 & 8.96 & 16.40 & 19.88 & 22.85 & 25.74 \\
Eccentric & 0.07 & 9.23 & 19.11 & 24.17 & 27.93 & 31.14 \\
Eccentric & 0.08 & 9.10 & 17.87 & 22.16 & 25.48 & 28.45 \\
Eccentric & 0.09 & 8.97 & 16.75 & 20.48 & 23.55 & 26.45 \\
\hline
\end{tabular}

Distribution of the heat transfer flux on the solid cylinder surface in the two types of concentric and eccentric porous layer was analyzed. Fig. 11 shows the heat flux distribution on the solid cylinder surface covered by an concentric and eccentric porous layer with the Reynolds number and Darcy number were considered 40 and $10^{-2}$, respectively. The average the heat transfer flux from the concentric porous layer state was obtained $35.29 \mathrm{w} / \mathrm{m}^{2}(\mathrm{~d}=0.07 \mathrm{~m}), 31.03$ $\mathrm{w} / \mathrm{m}^{2}(\mathrm{~d}=0.08 \mathrm{~m})$ and $28.79 \mathrm{w} / \mathrm{m}^{2}(\mathrm{~d}=0.09 \mathrm{~m})$. Also, for the eccentric porous layer state, the average heat transfer flux was achieved $39.52 \mathrm{w} / \mathrm{m}^{2}(\mathrm{~d}=0.07 \mathrm{~m}), 36.69 \mathrm{w} / \mathrm{m}^{2}(\mathrm{~d}=0.08 \mathrm{~m})$ and 34.92 $\mathrm{w} / \mathrm{m}^{2}(\mathrm{~d}=0.09 \mathrm{~m})$. According to the simulated results, with the increase in the porous layer diameter, the overall convection heat transfer rate was declined as well as with increasing the angle of direction fluid flow along the cylinder decrease gradually. 


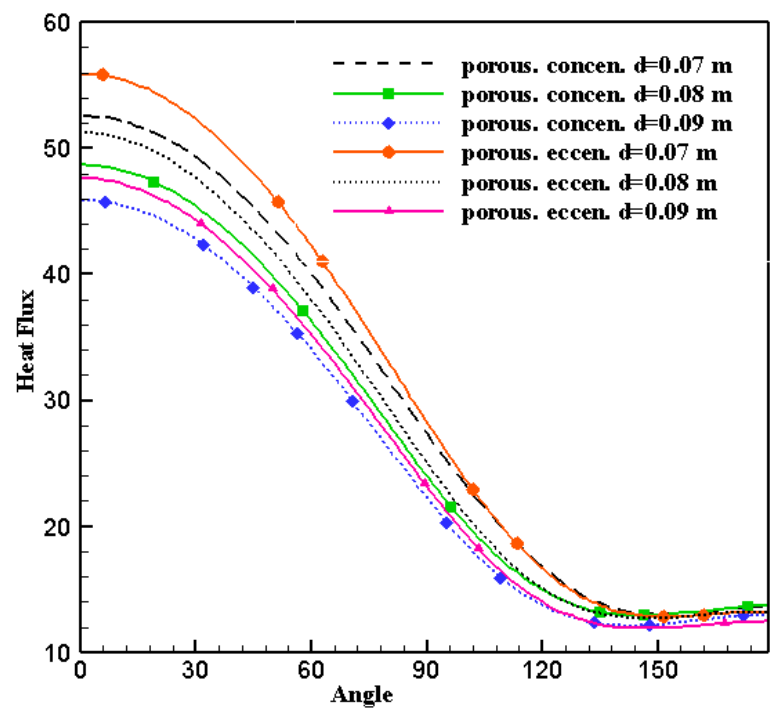

Fig . 11. Heat transfor flux from the solid cylinder surface.

The heat transfer flux distribution on the solid cylinder surface with different Reynolds number has been shown in Fig. 12. The solid cylinder surface was maintained at a fiexed temperature. The average heat flux of the cylinder surface to the fluid phase have shown in terms of various Reynolds numbers and Darcy numbers. According to the current numerical study, for an eccentric porous layer with the constant diameter $(\mathrm{d}=0.09 \mathrm{~m})$, it could be concluded that by increasing the Reynolds number, the heat flux of the cylinder was increased. Also, the average heat transfer flux surface ratio in $D a=10^{-2}$ greater than $D a=10^{-3}$ and $D a=10^{-6}$.

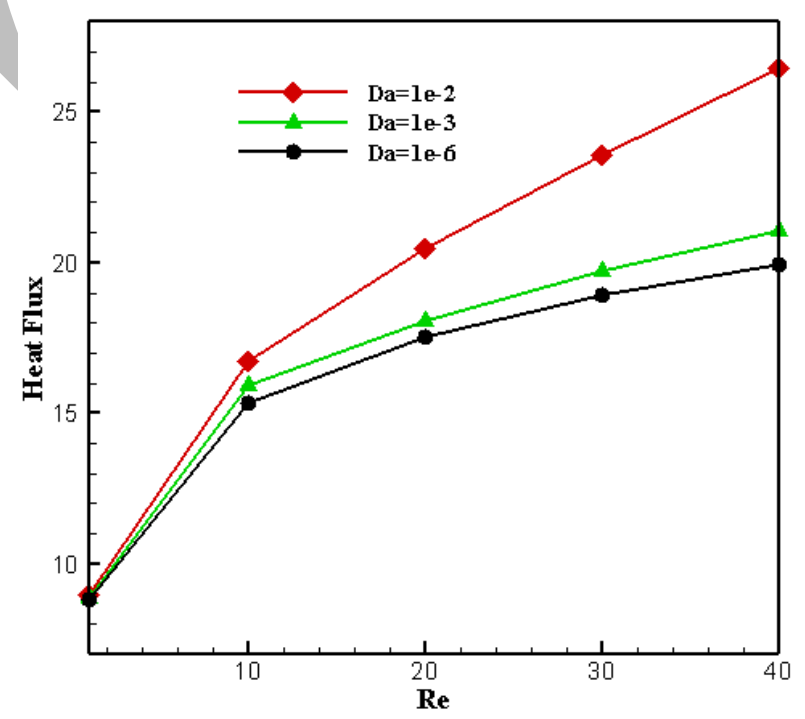

Fig . 12. Heat transfer flux on the solid cylinder surface with different Darcy and Reynolds numbers. 


\subsubsection{Nusselt Number}

Fig.13 shows the simulated results for the average Nusselt number with the variation of Darcy number in the various concentric and eccentric porous layer diameter.With the increase of the Darcy number, the average Nusselt number was raised. Similarity, as the porosity of the porous layer was increased, the fluid penetration and forced convection heat transfer were developed. Also, we found that for Darcy numbers greater than $10^{-4}$, the average Nusselt number was increased more fast. On the other hand, the effects of Darcy numbers higher than $10^{-4}$ was so much than the average Nusselt number lower than $10^{-4}$. Another remarkable point in Fig. 13 is that in a constant diameter and a fixed Darcy number, the effects of the eccentric porous layer on the Nusselt number would greater than that on the concentric porous layer. In the lower Darcy numbers, the porous layer behaves like that a solid rigid layer, this is because in the lower Darcy numbers, penetration was declined.

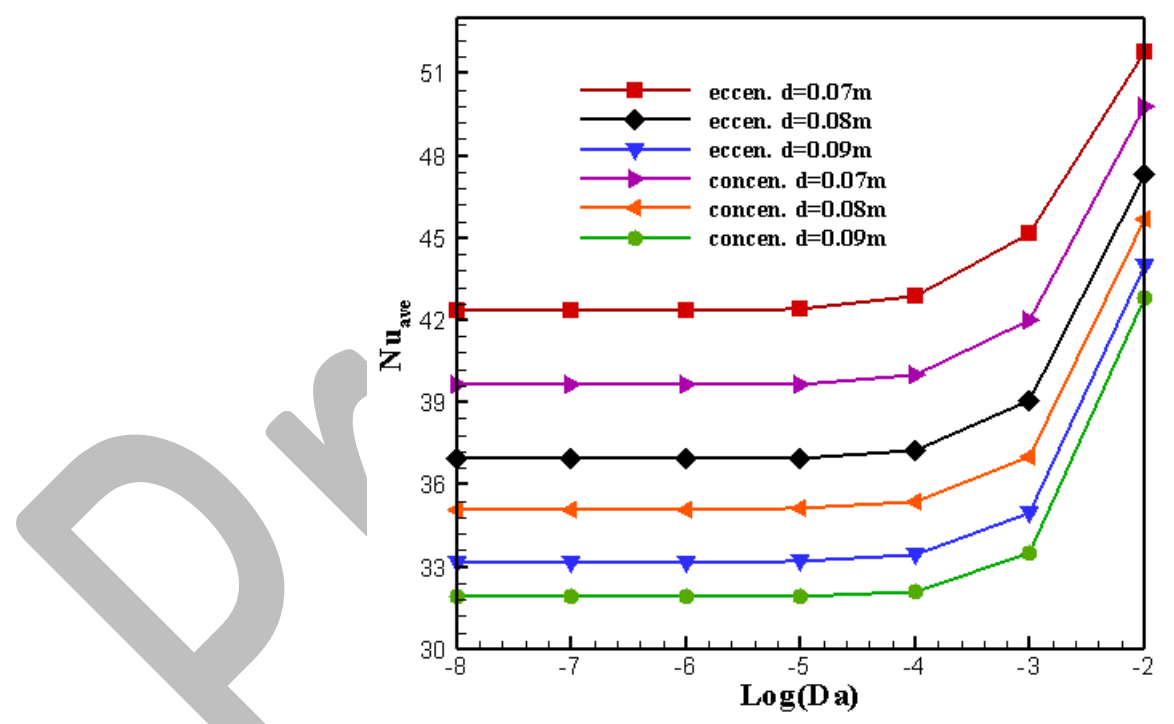

Fig . 13. Distribute the average Nusselt number on the solid cylinder surface with the various diameter of eccentric and concentric porous layer $\mathrm{Re}=40$.

According to Fig.14 regarding different values of Reynolds number for the porous layer, the numerical simulation of Nusselt number is shown in relation to the Darcy number. It can be seen in Fig.14 that with increasing the Darcy number associated with the eccentric porous layer with a diameter of 0.09 , the average Nusselt number was raised for all values related to the Reynolds parameters. As shown in Fig.14, the average Nusselt number for $D a \geq 10^{-4}$ was increased with a steeper slope. Also, the effects of Reynolds number on increasing Nusselt number was more 
significant for $D a \geq 10^{-4}$. In a fixed Darcy number, with the increase of the Reynolds number, the overall heat transfer rate from the surface to the fluid was greater.

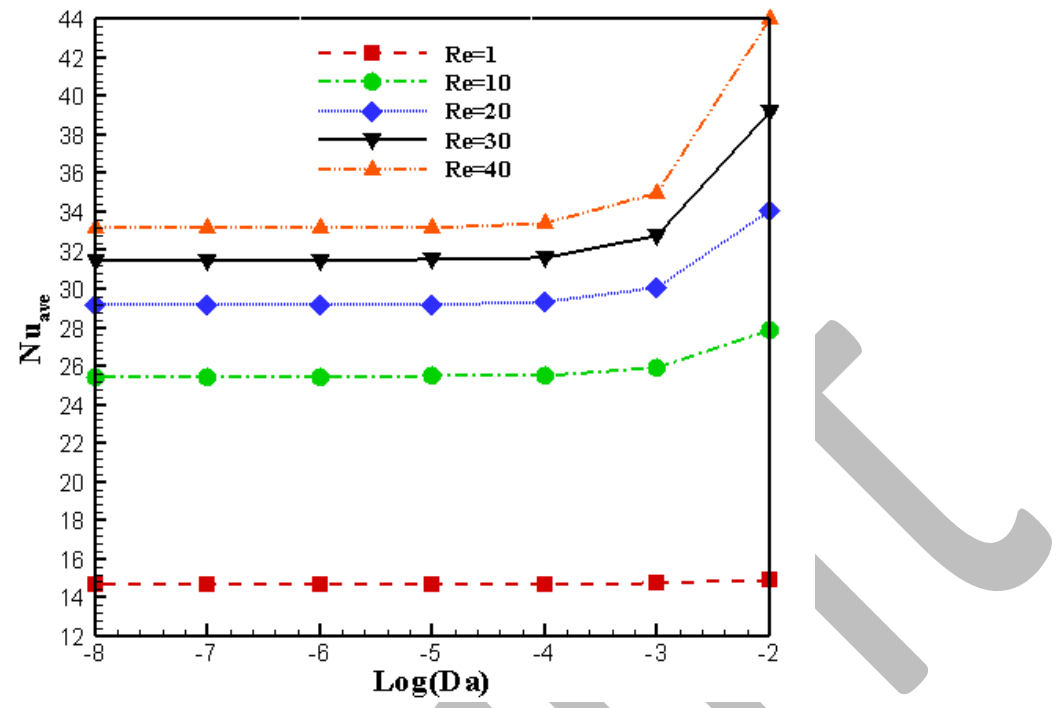

Fig. 14. Distribute the average Nusselt number on the cylinder surface to different Darcy numbers .

Fig. 15 shows the changes of the average Nusselt number relative to the Reynolds number changes for different values of the diameter of the porous layer in the concentric and eccentric states in constant Darcy number $\left(D a=10^{-2}\right)$. We have found with increasing Reynolds number, the mean is all different values of the concentric and eccentric diameter of the porous layers was raised. Another rasult we obtained, is that the effects of Reynolds number on increasing the average Nusselt number of the eccentric porous layer was higher than concentric porous layer. 


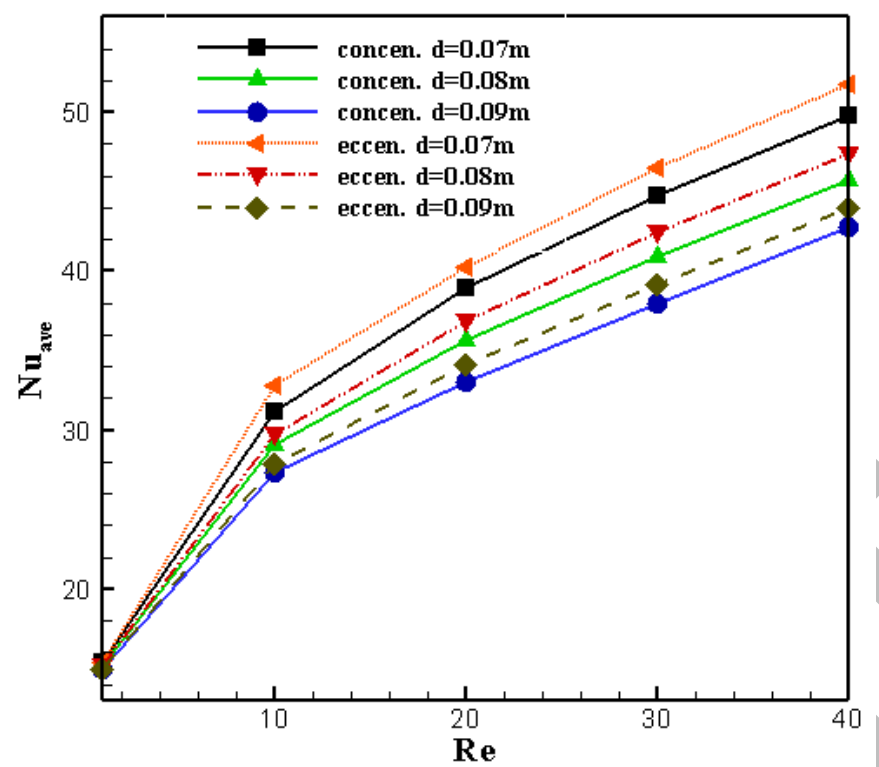

Fig. 15. The distribution of the average Nusselt number on the solid cylinder surface is different from the Reynolds numbers.

Figs. 16 and 17 show the variation of Nusselt number and the distribution of heat flux on the solid cylinder surface relative to the various eccentric porous layer diameter that was changing during simulation. This was the main reasult that we could obtained. the average Nusselt number and the distribution of the heat flux have grown whit increase the center of the eccentric porous layer from the center of the solid cylinder.It also states that whenever the eccentric porous layer was developed, force convection heat transfer rate into the fluid zone was increased. 

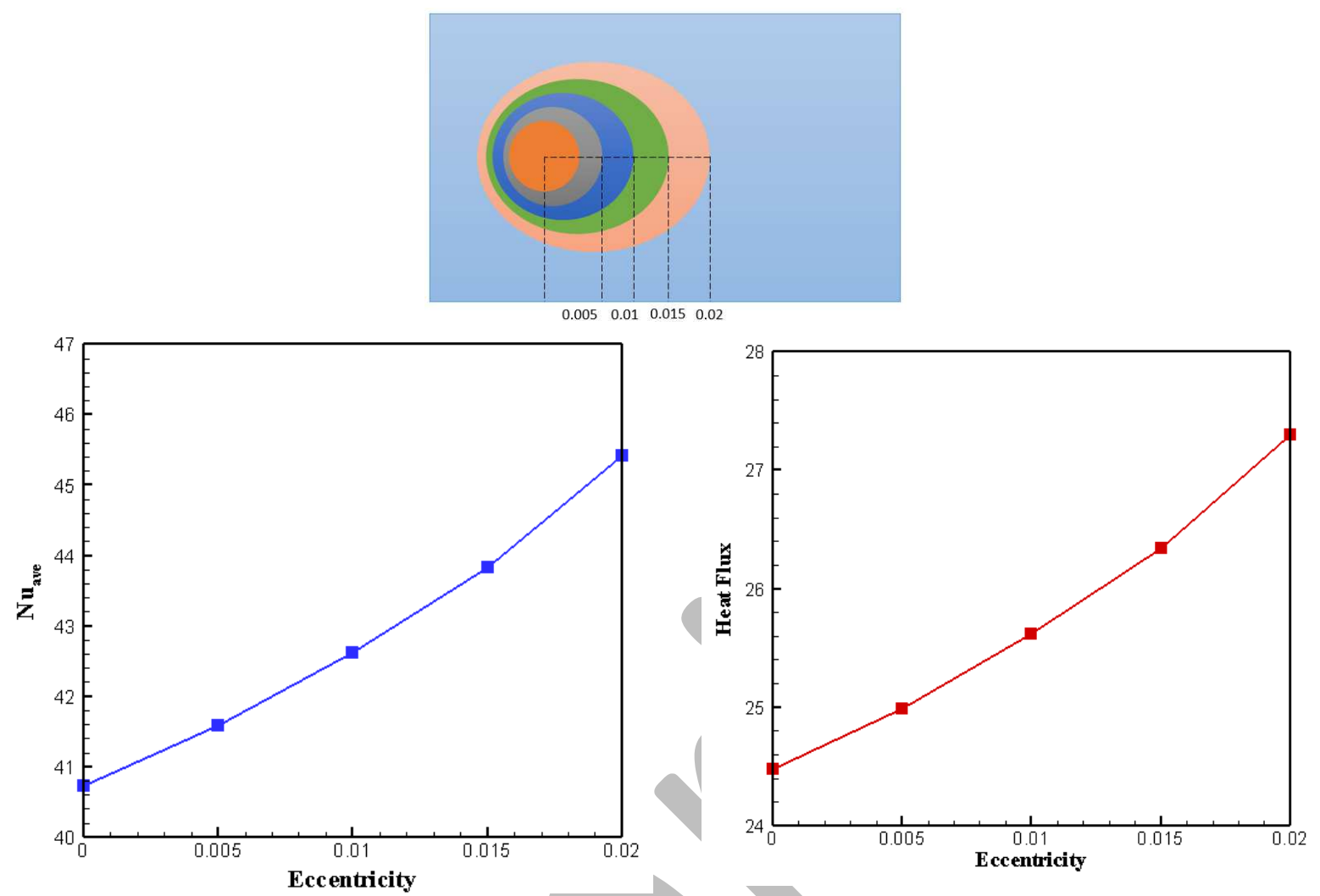

Fig - 16. Distribution of average Nusselt number on the cylinder surface with the various diameter of eccentric porous layer.

Fig. 17. Distribution of heat flux on the cylinder surface with the various diameter of eccentric porous layer.

\section{Conclusion}

In this study, the numerical simulationsbofbheat transfer and fluid flow around the twodimensional (2-D) cylinder wrapped around by concentric and eccentric porous layers were conducted. The effects of the different thickness of porous layer, Darcy number, Reynolds number, permeability, temperature and velocity (radial, tangential) distribution within the porous layer on the heat transfer and hydrodynamic flow were analyzed. In the first step of the study, we can find that The extent of the penetration of the fluid into the porous layer was increased with increasing the Darcy number. Also, the vortex formation length was developed with increasing the Reynolds number and decreasing Darcy number. Then, It could be concluded that by increasing the Darcy number at a constant Reynolds number and a constant diameter of porous layer, the heat flux rate passing through the surface of constant temperature of solid cylinder was 
raised. In the next stage of numerical investigation, The results have demonstrat obviously that in constant Reynolds numbers with the increase of Darcy number, the heat flux rate through the eccentric porous layer is more than the concentric porous layer. After that It could also be observed that with increasing the Darcy number $\left(\log (\boldsymbol{D} \boldsymbol{\alpha}) \geq \mathbf{1 0}^{\mathbf{- 4}}\right)$ and Reynolds number for all the different values of diameter of concentric and eccentric porous layer around the solid cylinder the average Nusselt number was raised. Ultimately, The study has shown that the average Nusselt number and the distribution of the heat flux have grown whit increase the center of the eccentric porous layer from the center of the solid cylinder.

\section{References}

[1] S. Bhattacharyya, S. Dhinakaran, A. Khalil, Fluid motion around and through a porous cylinder, Chem. Eng. Sci. 61 (13) (2006) 4451-4461.

[2] S. Bhattacharyya, A.K. Singh, Augmentation of heat transfer from a solid cylinder wrapped with a porous layer, Int J. Heat Mass Transfer 52 (7) (2009) 1991-2001.

[3] S. Rashidi, A. Tamayol, M.S. Valipour, N. Shokri, Fluid flow and forced convection heat transfer around a solid cylinder wrapped with a porous ring, Int. J. Heat Mass Transfer 63 (2013) 91-100.

[4] M.S. Valipour, A. Zare Ghadi, Numerical investigation of forced convective heat transfer around and through a porous circular cylinder with internal heat generation, J. Heat Transfer 134 (2012) 062601.

[5] F.M. Rong, Z.L. Guo, J.H. Lu, B.C. Shi, Numerical simulation of the flow around a porous covering square cylinder in a channel via lattice Boltzmann method, Int. J. Numer. Methods Fluids 65 (10) (2011) 1217-1230.

[6] N. Alvandifar, M. Saffar-Avval, E. Amani, Partially metal foam wrapped tube bundle as a novel generation of air cooled heat exchangers, Int J. Heat Mass Transfer 118 (2018) 171-181.

[7] M. Odabaee, K. Hooman, H. Gurgenci, Metal foam heat exchangers for heat transfer augmentation from a cylinder in cross-flow, Transp. Porous Media 86 (3) (2010) 911-923.

[8] N. Rahmati, Z. Mansoori, M. Saffar-Avval, G. Ahmadi, Numerical study of heat transfer in turbulent cross-flow over porous-coated cylinder. Am. Soc. Mech. Eng. FEDSM2017-69582 (2017) doi: 10.1115/FEDSM2017-69582.

[9] M. Ait Saada, S. Chikh, A. Campo, Natural convection around a horizontal solid cylinder wrapped with a layer of fibrous or porous material, Int. J. Heat Fluid Flow 28 (3) (2007) 483-495.

[10] G.F. Al-Sumaily, A. Nakayama, J. Sheridan, M.C. Thompson, The effect of porous media particle size on forced convection from a circular cylinder without assuming local thermal equilibrium between phases, Int J. Heat Mass Transfer 55 (2012) 3366-3378. 
[11] M.H. Pedras, M.J. de Lemos, Macroscopic turbulence modeling for incompressible flow through undeformable porous media, Int. J. Heat Mass Transfer 44 (2001) 1081-1093.

[12] K. Chen, L. Guo, X. Xie, W. Liu, Experimental investigation on enhanced thermal performance of staggered tube bundles wrapped with metallic foam, Int. J. Heat Mass Transfer 122 (2018) 459-468.

[13] K. Al-Salem, H.F. Oztop, S. Kiwan, Effects of porosity and thickness of porous sheets on heat transfer enhancement in a cross flow over heated cylinder, Int. Commun. Heat Mass Transfer 38 (9) (2011) 1279 1282.

[14] V.V. Calmidi, R.L. Mahajan, Forced convection in high porosity metal foams, J. Heat Transfer 122 (3) (2000) 557-565.

[15] C.Y. Zhao, T. Kim, T.J. Lu, H.P. Hodson, Thermal transport in high porosity cellular metal foams, J. Thermophys. Heat Transfer 18 (3) (2004) 309-317.

[16] E. Ebrahimi, Y. Amini, G. Imani, Heat transfer characteristics of a circular cylinder covered by a porous layer undergoing vortex-induced vibration. Int. J. Therm. Sci. 166 (2021) 106974.

[17] M.M. Keshtkar, M. Dadkhodazadeh, Thermal simulation of the symmetric and asymmetric arrangement of barriers on heat transfer enhancement in a porous gas heat exchanger, J. Therm. Sci. Eng. Appl. 10 (2018), 051001.

[18] Y.J. Zhuang, H.Z. Yu, Q.Y. Zhu, Experimental and numerical investigations on the flow around and through the fractal soft rocks with water vapor absorption, Int. J. Heat Mass Transfer 96 (2016) 413-429.

[19] H. Kaydani, A. Mohebbi, Experimental and numerical study of the onset of transient natural convection in a fractured porous medium, Transp. Porous Media 116 (2) (2017) 923-939.

[20] Y. Kaptan, E. Buyruk, A. Ecder, Numerical investigation of fouling on cross-flow heat exchanger tubes with conjugated heat transfer approach, Int. Commun. Heat Mass Transfer 35 (2008) 1153-1158.

\section{Acknowledgements}

The authors thank the chief of computer center of Department of Chemical Engineering, Shahid Bahonar University of Kerman, Kerman, Iran for supporting this work.

\section{Conflict of interest}

The authors declare no competing financial interest. 


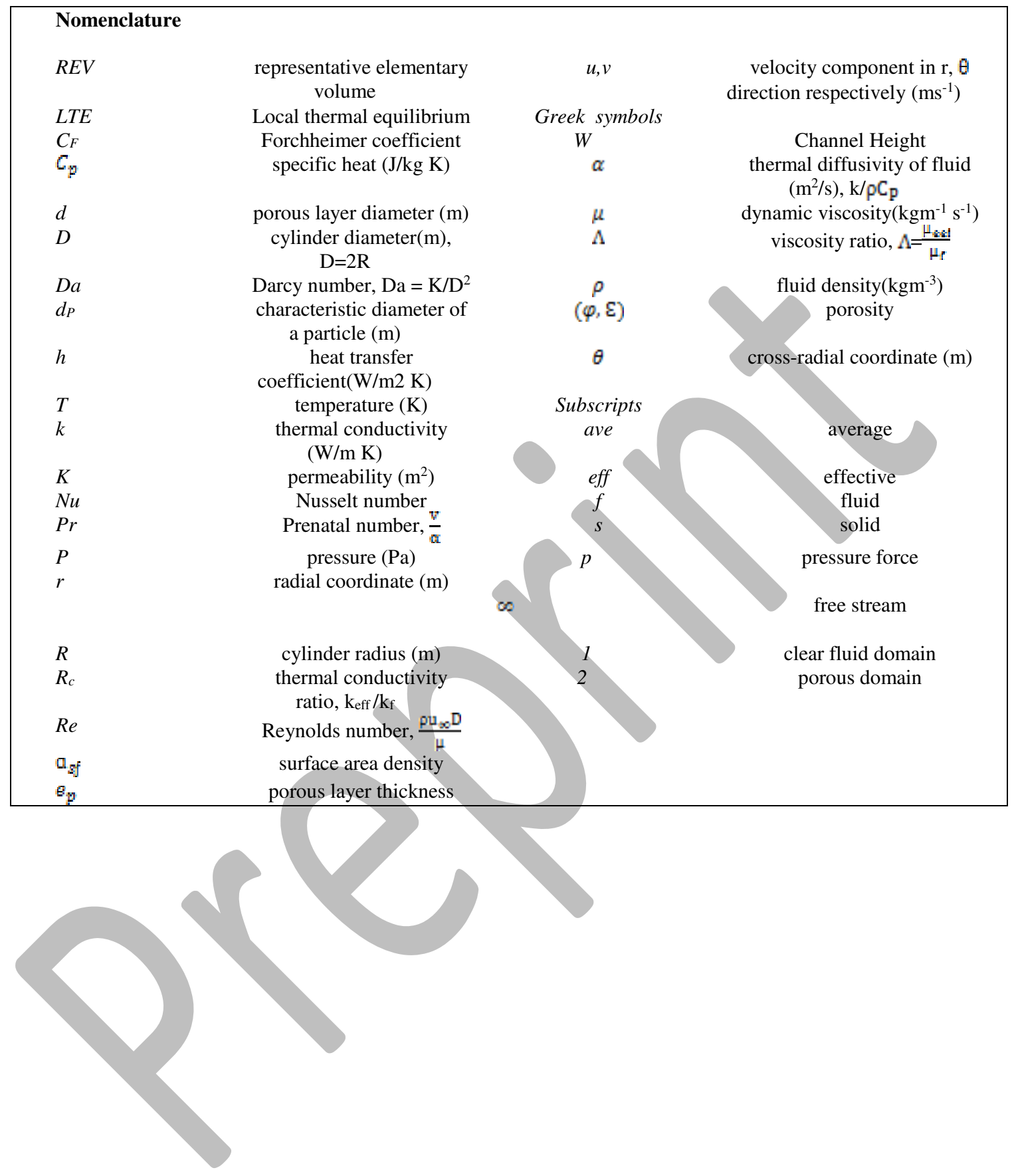

